\title{
Reconstruction of bi-dimensional images in Fourier-Transform Acousto-Optic Imaging
}

\author{
Maïmouna Bocoum ${ }^{1}$, Jean-Luc Gennisson ${ }^{2}$, Alexander A. Grabar ${ }^{3}$, François Ramaz ${ }^{1}$, And \\ JeAN-Michel TuALLE, ${ }^{4}$ \\ ${ }^{1}$ Institut Langevin, ESPCI Paris, PSL University, CNRS, 1 rue Jussieu, 75005 Paris, France \\ ${ }^{2}$ Université Paris-Saclay, CEA, CNRS, INSERM, BioMaps, Service Hospitalier Frédéric Joliot, 4 Place du Général Leclerc, 91401 Orsay, France \\ ${ }^{3}$ Physics Department, Uzhhorod National University, 88000 Uzhhorod, Ukraine \\ ${ }^{4}$ Laboratoire de Physique des Lasers, CNRS UMR 7538, Université Sorbonne Paris Nord, 99 avenue J.-B. Clément, 93430 Villetaneuse, France \\ *Corresponding author: tualle@univ-paris13.fr
}

We present a new method to perform acousto-optic imaging based on a spatio-temporal structuration of long-duration acoustic plane waves. This approach is particularly relevant when using detectors with long integration time. We show how it is possible to reconstruct an image by measuring its two-dimensional Fourier components. A proof of concept is presented using a photorefractive detection scheme, demonstrating equal performances to direct imaging. The overall acquisition time is compatible with medical monitoring applications. () 2020 Optical Society of America

http://dx.doi.org/10.1364/ao.XX.XXXXXX

Acousto-Optic Imaging (AOI) is an in-depth optical imaging technique of highly scattering media [1-13]. It relies on the detection of photons which have been tagged by user-controlled ballistic ultrasounds (US). For an optical source of high temporal coherence, the light exiting the medium is composed of multiple speckle grains with a random phase relation. As US are turned on, the speckle pattern undergoes a weakly contrasted modulation which in the spectral domain corresponds to a fraction of photons shifted by the US frequency. Various US spatio-temporal profiles can be used for tagging, among which pulsed [14] or continuous waves [2, 15], spatially focused [14] or unfocused plane waves $[16,17]$ and any of these combinations. For a given US profile, detection of tagged photons is then either performed from a single speckle grain $[2,14,15]$ or by summing the contribution from multiple speckle grains. This is done for instance using photorefractive holography $[8,10,18]$, spectralhole burning $[19,20]$ or camera-based detection [5], allowing to increase the signal-to-noise ratio (SNR) of an acquisition. Today, one of the main challenge of AOI is to demonstrate in-depth in vivo imaging. In this respect, it is crucial to work on the tagging-detection strategy which will allow $\mathrm{mm}$ spatial resolution with maximum SNR, while remaining insensitive to the speckle decorrelation induced by living tissues.

The most straightforward way to make an image is to record the photon flux tagged with a pulsed Focused Wave $(\mathrm{pFW})$ as it propagates along its collimated focal waist. A two-dimensional image is then obtained by performing a line-by-line scan of a chosen plane. To maintain the axial resolution in the millimeter range, the pulse should not exceed the $\mu$ s range in duration and its propagation should be sampled at a frequency higher than $1 \mathrm{MHz}$. To do so, photorefractive holographic-based detection is ideal because the signal is temporally sampled with a photodiode. However, the response time of photorefractive crystals with sufficient gain reported in the literature is typically greater than a millisecond $[8,10]$, which is not compatible with the decorrelation time inside living tissues ( 100-1000 $\mu$ s) [2124]. This is a major limitation for in vivo imaging. Spectral-hole burning $[19,20]$ is very promising because insensitive to speckle decorrelation, but in vivo imaging has yet not been demonstrated, in part because of the complexity and cumbersomeness of the cryogenic detection.

Long-exposure time camera-based detectors are a good alternatives to previously mentioned detection schemes because they can support short decorrelation time, provided that the latter remains greater than their exposure time $[9,25,26]$. The simultaneous detection of multiple speckle grains results from the superposition of the tagged photon field and an auxiliary reference field. Such detection requires however to adapt the US tagging strategy in order to account for their long integration time (typically $\geq \mu s$ ) with respect to US period. For instance, pFW imaging using CMOS detection was demonstrated with $5 \mathrm{~mm}$ resolution by setting the camera integration time to $3 \mu \mathrm{s}$ [9]. Increasing the exposure time by one or two orders of magnitude would significantly increase the SNR but drastically degrade the axial resolution. Alternatives have thus been proposed to recover longitudinal spatial resolution $[4,25,26]$ while maintaining long camera exposure times. All techniques rely on either phase or amplitude modulation of both the US pulse and reference arm. The resolution is no longer dependent on the pulse duration, but rather on the frequency bandwidth available to generate the modulation. For instance, periodic phase modulation was used to record the Fourier component of the image along US propagation direction [26]. This latter scheme is designated as Fourier Transform acousto-optic imaging (FT-AOI).

In the present paper, we generalize FT-AOI to the twodimensional case [27] using an acoustic transducer array, as 
opposed to a single mono-element. We will first recall the formalism used in $[26,28]$ and show how to tailor the emission of the transducer to easily adapt to this formalism [27]. To keep our approach as general as possible, we will theoretically present it for various detection schemes. The method will then be validated on experimental data using photorefractive holographic detection in order to provide simple comparison with pFW imaging.

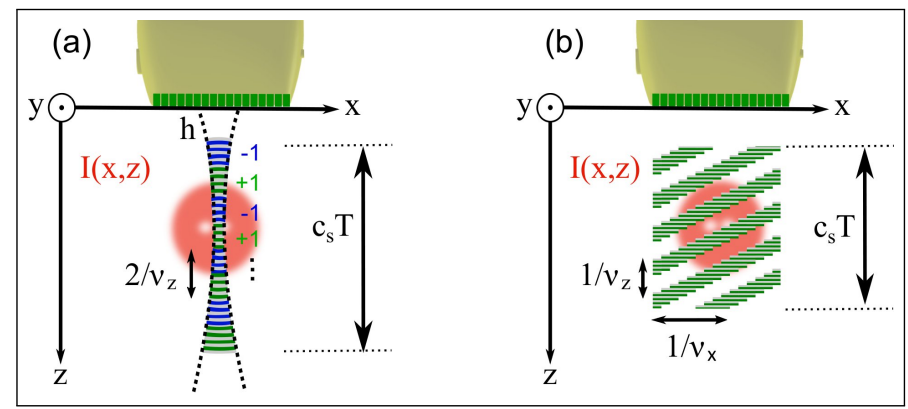

Fig. 1. Illustrations of (a) One-dimension FT-AOI case reported in $[26,28]$ and (b) Two-dimension FT-AOI investigated in the present work, with long pulses of duration $T$.

In one-dimension FT-AOI, the AC-voltage applied on a monoelement focusing transducer is phase-modulated as a function of time. As a result of US propagation, photons interact at all time with a pressure wave of complex amplitude $P(z, t)$, as illustrated in Fig 1(a). Note that the US carrier frequency is removed with the filtering of tagged photons, so we do not account for it within the scope of this article. Which ever the tagged photon detection, we define the signal $S_{i}$ recorded from a single measurement $i$ as:

$$
S_{i}=\int M_{i}(\vec{r}) I(\vec{r}) d^{3} \vec{r}
$$

where $M_{i}(\vec{r})$ is a complex tagging function associated to US propagation, and $I(\vec{r})$ is proportional to the local intensity of diffuse light, local absorption and scattering properties of the medium. Note that according to this definition, $I(\vec{r})$ is the image one would acquire performing pFW imaging. The tagging function $M_{i}(\vec{r})$ depends (i) on the spatio-temporal envelop of the US pulse and (ii) on the processing choice of the tagged signal.

In a camera-based detection, the tagged signal is modulated by a real function $h_{m}(t)$. For off-axis heterodyne holography [28] this is done on the auxilary reference field with an acousto-optic modulator. In heterodyne lock-in CMOS detection [26] the modulation is performed electronically on the detector chip. In both cases, the formalism is the same such that the tagging function for camera-based detection $M_{i}^{(a)}$ can be expressed as [28]:

$$
\left.M_{i}^{(a)}(\overrightarrow{(r})\right)=|\hat{P}|^{2}(\vec{r})
$$

where $\hat{P}$ is the virtual amplitude defined as:

$$
\hat{P}(\vec{r})=\frac{1}{T} \int_{0}^{T} h_{m}(t) P(\vec{r}, t) d t
$$

where $T$ is the integration time of the camera.

In one dimension, and neglecting diffraction of the US beam as it propagates, we can assume that $P(\vec{r}, t)=P_{0} h\left(t-c_{s}^{-1} z+\tau\right)$, where $h(t)$ is the pressure amplitude modulation function, $c_{S} \approx 1540 \mathrm{~m} / \mathrm{s}$ is the US velocity and $\tau$ a fixed delay. We now calculate $M_{i}^{(a)}$ using the $\mathrm{f} / 2$-periodic function $h$ plotted in Fig 2(a).
Imposing that $h_{m}(t)=\sin (\pi f t)$ as performed in [26] and setting the integration time such that $f T$ is an integer, we calculate using Eq 2 and Eq 3:

$$
M_{i}^{(a)}(z) \propto 1+\cos \left(2 \pi v_{z} z-\varphi\right)
$$

where $v_{z}=f / c_{s}$ is a spatial frequency, and $\varphi=2 \pi f \tau$ a phase offset which can be controlled independently of $v_{z}$ by changing the delay $\tau$. The calculated tagging function is the sum of a constant term and a sine function with a period half that of $P(z)$. Performing a linear combination of measurements $S_{i}$ acquired for $\varphi=0 ; \pi / 2 ; \pi$ and $3 \pi / 2$ we can numerically retrieve the complex Fourier component of the longitudinal profile corresponding to spatial frequency $v_{z}[17,26]$.

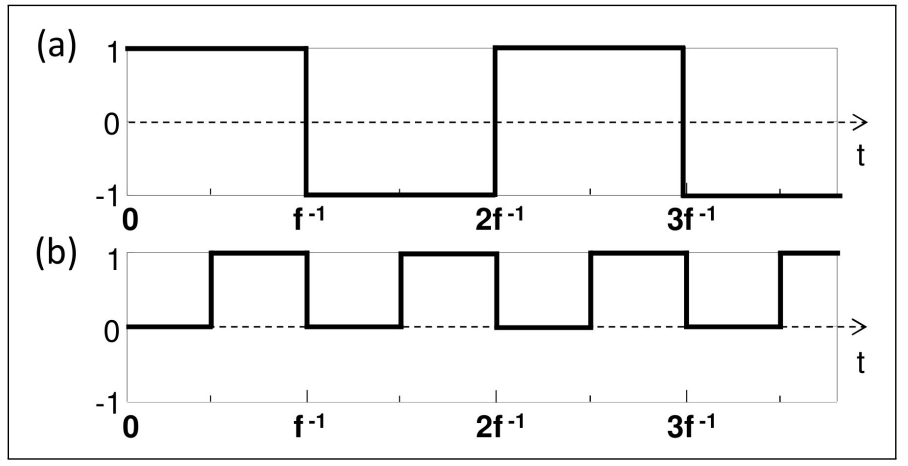

Fig. 2. Examples of possible modulation function $h(t)$ of the acoustic amplitude. (a): for camera-based detection; (b): for photorefractive holographic detection.

FT-AOI can also be implemented from a temporally sampled signal of tagged photon based on photorefractive holographicbased detection or spectral hole burning detection, where signal is sampled with a photodiode. In this case, the tagging function $M_{i}^{(b)}$ is obtained by post-prossessing of the acquired temporal trace, which allows to choose a complex function for $h_{m}$ before performing a numerical integration over time $T$. The expression of $M_{i}^{(b)}$ is given by:

$$
M_{i}^{(b)}(\vec{r})=\frac{1}{T} \int_{0}^{T} h_{m}(t)|P|^{2}(\vec{r}, t) d t
$$

In this case, the 4-phase measurement is prevented by replacing the previous real function by $h_{m}(t)=\exp (-2 \pi j f t)$, while imposing that $h(t)$ oscillates between 1 and 0 at a frequency $f$ as shown in Fig. 2(b). This corresponds to an amplitude rather than phase modulation of the pressure field. This is in particular what will be presented in the following experimental results, where detection is based on photorefractive holography. In this case, we easily calculate:

$$
M_{i}^{(b)}(z) \propto j \exp \left(-2 \pi j v_{z} z+j \varphi\right)
$$

Such a tagging function directly provides Fourier component of the longitudinal profile for $\varphi=-\pi / 2$.

To generalize this to the two-dimensional case, we propose to use a long-duration US modulated plane wave (as opposed to focused), propagating along $z$ direction. The substitution $h(z, t) \rightarrow h(x, z, t)$ is possible by controlling independently the temporal US emission at each position $x$. The amplitude modulation at position $x$ is set to $h\left[t+\tau_{m}(x)\right]$. The delay $\tau_{m}(x)$ depends linearly on the transverse position $x$ such that: 


$$
\tau_{m}(x)=\tau-\frac{v_{x} x}{f}=\tau-\frac{v_{x} x}{v_{z} c_{s}}
$$

Still neglecting diffraction effects, we now have $P(x, z, t)=$ $P_{0} h\left[t-c_{s}^{-1} z+\tau_{m}(x)\right]$ as illutrated in Fig $1(\mathrm{~b})$. The twodimensional tagging functions simply result from the substitution $\varphi=2 \pi f \tau \rightarrow 2 \pi f \tau_{m}(x)=\varphi-2 \pi v_{x} x$ in respectively Eq. 4 and 6. Using the previous functions $h(t)$ plotted respectively in Fig 2(a) and Fig 2(b) and the respective demodulation functions $h_{m}(t)=\sin (\pi f t)$ and $h_{m}(t)=\exp (-2 \pi j f t)$, we calculate the following two-dimensional tagging functions:

$$
\left\{\begin{array}{l}
M^{(a)}(x, z) \propto 1+\cos \left(2 \pi v_{x} x+2 \pi v_{z} z-\varphi\right) \\
M^{(b)}(x, z) \propto j \exp \left(-2 \pi j v_{x} x-2 \pi j v_{z} z+j \varphi\right)
\end{array}\right.
$$

This constitutes a 2D generalization of previous tagging functions. As a result, 2D Fourier components of the AO image in the $(x, z)$-plane are obtained with a 4-phase measurement for camera based-detection and in a single measurement for temporally sampled AO signals. Let us note that the theoretical expressions in Eqs. 8 exhibit a sinusoidal behavior, thanks to the filtering of the square modulation by temporal averaging in Eqs. 3 and 5. As a result, accurate extraction of Fourier components in the whole Fourier plane is possible, as opposed to previous work reported on unfocused US waves. In [16] for instance, tilted plane waves are emitted with limited angular span such that many Fourier components can not be measured. By structuring these waves laterally [17], more Fourier components can be reached but an error of reconstruction is made for modulations that aren't purely sinusoidal. Since US transducer arrays do not generally allow for smooth modulation of the US wave, the harmonic content of the modulation leads to image degradation [17]. This effect is absent in our present approach thanks to the above-mentioned filtering. Furthermore, both of these methods work in the short-pulse regime, contrary to what we propose here.

We present here the experimental validation of 2D FT-AOI using photorefractive holographic-based detection. This choice of detection might seem peculiar given that the imaging approach presented here is particularly interesting for camera-based detection, potential candidates for in vivo imaging applications. From a fundamental point of view, this choice of detection is justified because it allows direct comparison with a pFW imaging, and is much simpler to implement.

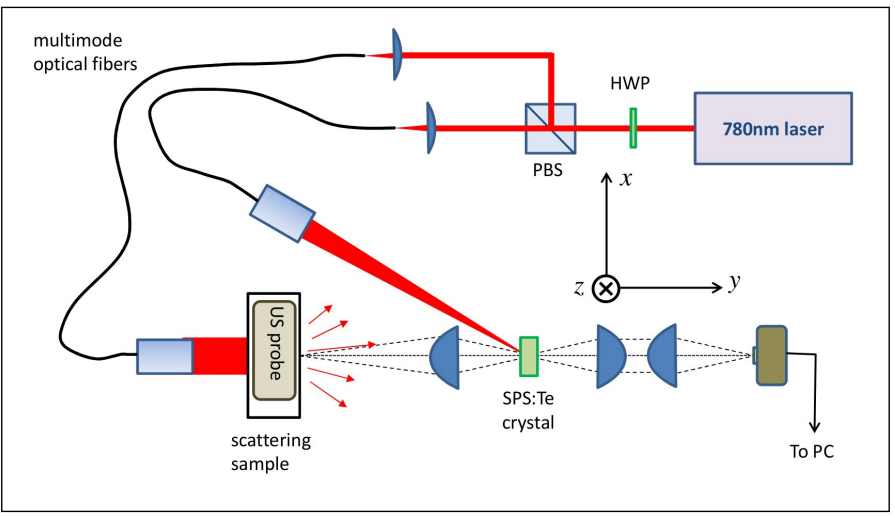

Fig. 3. Experimental setup. (HWP): Half Wave Plate. (PBS): Polarising Beam Splitter.
A scheme of the experiment is shown in Fig. 3. The sample is an Agar gel matrix with dimensions $H \times L \times W=$ $5 \times 5 \times 2.5 \mathrm{~cm}^{3}$ with $17 \%$ concentration of intralipid. The gel reduced scattering coefficient $\mu_{s}^{\prime} \approx 10 \mathrm{~cm}^{-1}$ is representative of biological tissues [29]. Two absorbing inclusions of respectively $2 \mathrm{~mm}$ and $3 \mathrm{~mm}$ inner diameter and $5 \mathrm{~mm}$ length (along the transverse $y$ direction), with a separation of $4 \mathrm{~mm}$ between their centers, were embedded in the middle of the sample thickness (y direction) at $\approx 13 \mathrm{~mm}$ below the upper face. A photograph of these inclusions is shown in Fig. 4(a) before being recovered by another layer of scattering gel. The sample is illuminated with a single-longitudinal mode laser centered at $780 \mathrm{~nm}$. After the tapered amplifier (MOPA, Sacher Lasertechnik $\mathrm{GmBH}$ ), the incident power is about $\approx 500 \mathrm{~mW}$ for a beam diameter of $\approx 7 \mathrm{~mm}$. The tagged light is collected in transmission through the sample with a photorefractive-based detection scheme (SPS crystal doped with Te 1\%) described elsewhere $[8,10,13]$. The tagged photon flux is recorded with a biased Si-photodiode (Thorlabs Det36A-10 MHz bandwidth, $13 \mathrm{~mm}^{2}$ surface) coupled to a transimpedance amplifier (Femto GmBh), and sampled at $10 \mathrm{MHz}$ with an acquisition board (Gage Digitizer). Our transducer array is a commercial US probe (SL10-2, Supersonic Imagine, Aix en Provence, France) with a fixed focal length in y-direction of $35 \mathrm{~mm}$. The probe has 192 piezo-elements of $200 \mu \mathrm{m}$ width, resulting in a total width of $38.4 \mathrm{~mm}$. We positioned it on the top of the sample, above a medical gel, and each piezo-element of the array is arbitrarily addressed to generate the structured acoustic plane wave. In particular, each element emits $100 \mu \mathrm{s}-$ long US pulses centered at $3 \mathrm{MHz}$, modulated with the function $h$ plotted in Fig. 2(b), delayed according to the law given in $\mathrm{Eq} 7$, and at a repetition rate of $1 \mathrm{kHz}$. Note that the size of the piezo-elements is smaller than the US wavelength, ensuring a good sampling of the US. At all time, the US wavefront is encoded parallel to the emission plane so that US propagate in the $\mathrm{z}$ direction. With an excitation voltage of $15 \mathrm{~V}$ for the piezoelements, the maximal pressure of the US wave was measured to be $\approx 300 \mathrm{kPa}$. Such a pressure corresponds to a US peak intensity of $\approx 3 \mathrm{~W} / \mathrm{cm}^{2}$ and, taking the duty cycle into account, to an average intensity of $\approx 150 \mathrm{~mW} / \mathrm{cm}^{2}$ far below biomedical norms $\left(720 \mathrm{~mW} / \mathrm{cm}^{2}\right)[30]$.

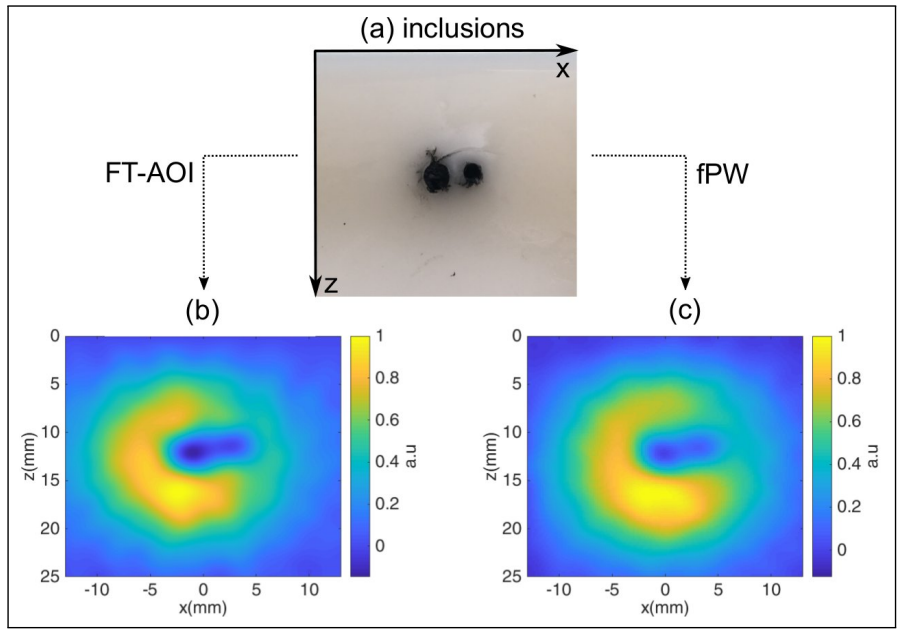

Fig. 4. (a): pictures of the inclusions, before being recovered by another layer of scattering gel; (b) normalized image obtained from FT-AOI; (c) normalized filtered image recorded with pFW. 
To make an image, we record successive traces at a $1 \mathrm{kHz}$ repetition rate. Between each of the traces, $\left(v_{x}, v_{z}\right)$ values are changed so as to map the Fourier plane of the image. Temporal traces are thus acquired for $v_{x}=m v_{x 0}$ and $v_{z}=n v_{z 0}$, where $v_{x 0}$ and $v_{z 0}$ are the fundamental frequencies. They are set to $v_{x 0}=0.026 \mathrm{~mm}^{-1}$ and $v_{z 0}=1 /\left(c_{s} T_{0}\right) \approx 0.033 \mathrm{~mm}^{-1}$, where $T_{0}=20 \mu s$ is the fundamental temporal modulation. This corresponds to an image field of view of $30 \mathrm{~mm}$ in direction $z$ and $38.4 \mathrm{~mm}$ in direction $x$. We chose $m$ and $n$ as integers such that $-10 \leq m \leq 10$, and $1 \leq n \leq 10$. This adds up to 210 Fourier components in total, and the full acquisition was repeated 10 times for signal averaging. This therefore corresponds to an overall acquisition time of 2.1s. To reconstruct an image, each averaged trace is multiplied by $h_{m}(t)=\exp (-2 \pi j f t)$, where $f=c_{s} v_{z}$. Each $\left(v_{x}, v_{z}\right)$-Fourier component is then calculated by integrating over time T. We arbitrarily set that value to $T=3 T_{0}=60 \mu \mathrm{s}$ so as to mimic an integration time typical of camera-based detectors compatible with in vivo decorrelation time scales. The components at $v_{z}=0$ are extrapolated from the hypothesis that the AO image cancels close to the surface. The reconstructed image is shown on Fig 4(b) where the two inclusions can be distinguished. We then wish to compare this image to that obtained using $\mathrm{pFW}$ waves limited to the same Fourier components. This reference image is performed by leaving the probe at the same position and scanning the image plane over 143 positions along the $x$ axis with a focused pulse of 3 acoustic cycle in duration centered at $3 \mathrm{MHz}$. We then applied a low-pass filter on the image so as to match the Fourier plane explored in our 2D-FT imaging setup. The result is represented in Fig 4(c), and is very similar to our previous image. This demonstrates that within the explored Fourier region, the image quality of both techniques are equivalent. At last, we would like to emphasize that for the pFW image, the nominal pressure was increased to $40 \mathrm{~V}$, so that the acoustic peak intensity can exceed $200 \mathrm{~W} / \mathrm{cm}^{2}$ at the focal point. In addition, each line was averaged 500 times leading to an overall acquisition time of $71.5 \mathrm{~s}$, that is to say 34 times higher than with FT-AOI. Although these parameters are only indicative, they illustrate the potential of FT-AOI for real life applications.

In conclusion, we have provided the first proof of concept for bi-dimensional Fourier-Transform Acousto-Optic Imaging. This method, which is an extension to that presented in [26], relies on the spatio-temporal modulation of an acoustic plane wave to extract the Fourier components of the acousto-optic image. It is based on a simple model where US diffraction is neglected. This model is validated by our experimental results, where the image of two inclusions obtained with FT-AOI compares well with that obtained with standard $\mathrm{pFW}$ for a given Fourier domain of exploration. So far, our result is important because 2D FTAOI imaging relies on the use of long-duration acoustic pulses compatible with camera-based detection setups, potential candidates for in vivo imaging. In addition, plane waves and long pulses allow to reduce the constraint on the acoustic peak-power in order to stay below biomedical norms. In future work we plan to reproduce these results using of an off-axis heterodyne holography detection schemes so as to pave the way towards in vivo imaging.

\section{FUNDING INFORMATION}

Plan Cancer ITMO-INSERM 2014-2019 (MALT-C16027HS); Défi Imag'In-CNRS (MALT); French LABEX WIFI (ANR-10-LABX-24 and ANR-10-IDEX-0001-02 PSL).

\section{DISCLOSURES}

The authors declare no conflicts of interest.

\section{REFERENCES}

1. J. Gunther and S. Andersson-Engels, Front. Optoelectronics 10, 211 (2017).

2. W. Leutz and G. Maret, Phys. B: Condens. Matter 204, 14 (1995).

3. M. Kempe, M. Larionov, D. Zaslavsky, and A. Z. Genack, J. Opt. Soc. Am. A 14, 1151 (1997).

4. L. V. Wang and G. Ku, Opt. Lett. 23, 975 (1998).

5. S. Lévêque, A. C. Boccara, M. Lebec, and H. Saint-Jalmes, Opt. Lett. 24, 181 (1999).

6. L. V. Wang, Opt. Lett. 26, 1191 (2001).

7. S. Sakadžić and L. V. Wang, Opt. Lett. 29, 2770 (2004).

8. F. Ramaz, B. C. Forget, M. Atlan, A. C. Boccara, M. Gross, P. Delaye, and G. Roosen, Opt. Express 12, 5469 (2004).

9. M. Atlan, B. C. Forget, F. Ramaz, A. C. Boccara, and M. Gross, Opt. Lett. 30, 1360 (2005).

10. S. Farahi, G. Montemezzani, A. A. Grabar, J.-P. Huignard, and F. Ramaz, Opt. Lett. 35, 1798 (2010).

11. N. T. Huynh, D. He, B. R. Hayes-Gill, J. A. Crowe, J. G. Walker, M. L. Mather, F. R. Rose, S. P. Morgan, N. G. Parker, and M. J. Povey, J. Biomed. Opt. 17, 1 (2012).

12. H. Ruan, M. L. Mather, and S. P. Morgan, Opt. Lett. 37, 1658 (2012).

13. J.-B. Laudereau, E. B. à La Guillaume, V. Servois, P. Mariani, A. A. Grabar, M. Tanter, J.-L. Gennisson, and F. Ramaz, J. Biophotonics 8 , 429 (2015).

14. F. A. Marks, H. W. Tomlinson, and G. W. Brooksby, "Comprehensive approach to breast cancer detection using light: photon localization by ultrasound modulation and tissue characterization by spectral discrimination," in Photon Migration and Imaging in Random Media and Tissues, , vol. 1888 (International Society for Optics and Photonics, 1993), pp. 500-510.

15. L. Wang, S. L. Jacques, and X. Zhao, Opt. letters 20, 629 (1995).

16. J.-B. Laudereau, A. A. Grabar, M. Tanter, J.-L. Gennisson, and F. Ramaz, Opt. Express 24, 3774 (2016).

17. M. Bocoum, J.-L. Gennisson, J.-B. Laudereau, A. Louchet-Chauvet, J.-M. Tualle, and F. Ramaz, Appl. Opt. 58, 1933 (2019).

18. T. W. Murray, L. Sui, G. Maguluri, R. A. Roy, A. Nieva, F. Blonigen, and C. A. DiMarzio, Opt. letters 29, 2509 (2004).

19. Y. Li, H. Zhang, C. Kim, K. H. Wagner, P. Hemmer, and L. V. Wang, Appl. Phys. Lett. 93, 011111 (2008).

20. C. Venet, M. Bocoum, J.-B. Laudereau, T. Chanelière, F. Ramaz, and A. Louchet-Chauvet, Opt. letters 43, 3993 (2018).

21. M. Gross, P. Goy, B. Forget, M. Atlan, F. Ramaz, A. Boccara, and A. K. Dunn, Opt. letters 30, 1357 (2005).

22. A. Lev and B. Sfez, JOSA A 20, 2347 (2003).

23. Y. Liu, P. Lai, C. Ma, X. Xu, A. A. Grabar, and L. V. Wang, Nat. communications 6, 1 (2015).

24. M. M. Qureshi, J. Brake, H.-J. Jeon, H. Ruan, Y. Liu, A. M. Safi, T. J. Eom, C. Yang, and E. Chung, Biomed. optics express 8, 4855 (2017).

25. M. Lesaffre, S. Farahi, M. Gross, P. Delaye, A. Boccara, and F. Ramaz, Opt. Express 17, 18211 (2009).

26. K. Barjean, K. Contreras, J.-B. Laudereau, Éric Tinet, D. Ettori, F. Ramaz, and J.-M. Tualle, Opt. Lett. 40, 705 (2015).

27. J.-M. Tualle, F. Ramaz, J.-L. Gennisson, and M. Bocoum, patent application FR1856378 (2018).

28. K. Barjean, F. Ramaz, and J.-M. Tualle, J. Opt. Soc. Am. A 33, 854 (2016).

29. S. L. Jacques, Phys. Medicine Biol. 58, R37 (2013).

30. R. Phillips and G. Harris, "Information for manufacturers seeking marketing clearance of diagnostic ultrasound systems and transducers," Tech. rep., Food and Drug Administration (2008). 


\section{FULL REFERENCES}

1. J. Gunther and S. Andersson-Engels, "Review of current methods of acousto-optical tomography for biomedical applications," Front. Optoelectronics 10, 211-238 (2017).

2. W. Leutz and G. Maret, "Ultrasonic modulation of multiply scattered light," Phys. B: Condens. Matter 204, 14 - 19 (1995).

3. M. Kempe, M. Larionov, D. Zaslavsky, and A. Z. Genack, "Acoustooptic tomography with multiply scattered light," J. Opt. Soc. Am. A 14, 1151-1158 (1997).

4. L. V. Wang and G. Ku, "Frequency-swept ultrasound-modulated optical tomography of scattering media," Opt. Lett. 23, 975-977 (1998).

5. S. Lévêque, A. C. Boccara, M. Lebec, and H. Saint-Jalmes, "Ultrasonic tagging of photon paths in scattering media:?parallel speckle modulation processing," Opt. Lett. 24, 181-183 (1999).

6. L. V. Wang, "Mechanisms of ultrasonic modulation of multiply scattered coherent light: a monte carlo model," Opt. Lett. 26, 1191-1193 (2001).

7. S. Sakadžić and L. V. Wang, "High-resolution ultrasound-modulated optical tomography in biological tissues," Opt. Lett. 29, 2770-2772 (2004).

8. F. Ramaz, B. C. Forget, M. Atlan, A. C. Boccara, M. Gross, P. Delaye, and G. Roosen, "Photorefractive detection of tagged photons in ultrasound modulated optical tomography of thick biological tissues," Opt. Express 12, 5469-5474 (2004).

9. M. Atlan, B. C. Forget, F. Ramaz, A. C. Boccara, and M. Gross, "Pulsed acousto-optic imaging in dynamic scattering media with heterodyne parallel speckle detection," Opt. Lett. 30, 1360-1362 (2005).

10. S. Farahi, G. Montemezzani, A. A. Grabar, J.-P. Huignard, and F. Ramaz, "Photorefractive acousto-optic imaging in thick scattering media at $790 \mathrm{~nm}$ with a sn2p2s6:te crystal," Opt. Lett. 35, 1798-1800 (2010).

11. N. T. Huynh, D. He, B. R. Hayes-Gill, J. A. Crowe, J. G. Walker, M. L. Mather, F. R. Rose, S. P. Morgan, N. G. Parker, and M. J. Povey, "Application of a maximum likelihood algorithm to ultrasound modulated optical tomography," J. Biomed. Opt. 17, 1 - 13 (2012).

12. H. Ruan, M. L. Mather, and S. P. Morgan, "Pulse inversion ultrasound modulated optical tomography," Opt. Lett. 37, 1658-1660 (2012).

13. J.-B. Laudereau, E. B. à La Guillaume, V. Servois, P. Mariani, A. A. Grabar, M. Tanter, J.-L. Gennisson, and F. Ramaz, "Multi-modal acousto-optic/ultrasound imaging of ex vivo liver tumors at $790 \mathrm{~nm}$ using a sn2p2s 6 wavefront adaptive holographic setup," J. Biophotonics 8, 429-436 (2015).

14. F. A. Marks, H. W. Tomlinson, and G. W. Brooksby, "Comprehensive approach to breast cancer detection using light: photon localization by ultrasound modulation and tissue characterization by spectral discrimination," in Photon Migration and Imaging in Random Media and Tissues, vol. 1888 (International Society for Optics and Photonics, 1993), pp. 500-510.

15. L. Wang, S. L. Jacques, and X. Zhao, "Continuous-wave ultrasonic modulation of scattered laser light to image objects in turbid media," Opt. letters 20, 629-631 (1995).

16. J.-B. Laudereau, A. A. Grabar, M. Tanter, J.-L. Gennisson, and F. Ramaz, "Ultrafast acousto-optic imaging with ultrasonic plane waves," Opt. Express 24, 3774-3789 (2016).

17. M. Bocoum, J.-L. Gennisson, J.-B. Laudereau, A. Louchet-Chauvet, J.-M. Tualle, and F. Ramaz, "Structured ultrasound-modulated optical tomography," Appl. Opt. 58, 1933-1940 (2019).

18. T. W. Murray, L. Sui, G. Maguluri, R. A. Roy, A. Nieva, F. Blonigen, and C. A. DiMarzio, "Detection of ultrasound-modulated photons in diffuse media using the photorefractive effect," Opt. letters 29, 2509-2511 (2004).

19. Y. Li, H. Zhang, C. Kim, K. H. Wagner, P. Hemmer, and L. V. Wang, "Pulsed ultrasound-modulated optical tomography using spectral-hole burning as a narrowband spectral filter," Appl. Phys. Lett. 93, 011111 (2008).

20. C. Venet, M. Bocoum, J.-B. Laudereau, T. Chanelière, F. Ramaz, and A. Louchet-Chauvet, "Ultrasound-modulated optical tomography in scattering media: flux filtering based on persistent spectral hole burning in the optical diagnosis window," Opt. letters 43, 3993-3996 (2018).

21. M. Gross, P. Goy, B. Forget, M. Atlan, F. Ramaz, A. Boccara, and A. K.
Dunn, "Heterodyne detection of multiply scattered monochromatic light with a multipixel detector," Opt. letters 30, 1357-1359 (2005).

22. A. Lev and B. Sfez, "In vivo demonstration of the ultrasound-modulated light technique," JOSA A 20, 2347-2354 (2003).

23. Y. Liu, P. Lai, C. Ma, X. Xu, A. A. Grabar, and L. V. Wang, "Optical focusing deep inside dynamic scattering media with near-infrared timereversed ultrasonically encoded (true) light," Nat. communications 6 , 1-9 (2015).

24. M. M. Qureshi, J. Brake, H.-J. Jeon, H. Ruan, Y. Liu, A. M. Safi, T. J. Eom, C. Yang, and E. Chung, "In vivo study of optical speckle decorrelation time across depths in the mouse brain," Biomed. optics express 8, 4855-4864 (2017)

25. M. Lesaffre, S. Farahi, M. Gross, P. Delaye, A. Boccara, and F. Ramaz, "Acousto-optical coherence tomography using random phase jumps on ultrasound and light," Opt. Express 17, 18211-18218 (2009).

26. K. Barjean, K. Contreras, J.-B. Laudereau, Éric Tinet, D. Ettori, F. Ramaz, and J.-M. Tualle, "Fourier transform acousto-optic imaging with a custom-designed cmos smart-pixels array," Opt. Lett. 40, 705-708 (2015).

27. J.-M. Tualle, F. Ramaz, J.-L. Gennisson, and M. Bocoum, "Procédé d'imagerie acousto-optique par reconstruction de fourier en utilisant une onde plane comme onde porteuse de l'onde ultrasonore," patent application FR1856378 (2018).

28. K. Barjean, F. Ramaz, and J.-M. Tualle, "Theoretical study of fouriertransform acousto-optic imaging," J. Opt. Soc. Am. A 33, 854-862 (2016).

29. S. L. Jacques, "Optical properties of biological tissues: a review," Phys. Medicine Biol. 58, R37-R61 (2013).

30. R. Phillips and G. Harris, "Information for manufacturers seeking marketing clearance of diagnostic ultrasound systems and transducers," Tech. rep., Food and Drug Administration (2008). 